\title{
FACTORES DE PERSONALIDAD Y ESTRATEGIAS DE AFRONTAMIENTO EN MUJERES: RELACIONES Y DIFERENCIAS
}

\author{
Consuelo Morán Astorga \\ Universidad de León \\ mcmora@unileon.es \\ Juana María Torres Calles \\ Orientadora Secundaria. Jaén
}

Fecha de Recepción: 8 Marzo 2019

Fecha de Admisión: 30 Abril 2019

\section{RESUMEN}

En este estudio deseamos averiguar si las estrategias de afrontamiento formulados por Lazarus y Folkman (1986) se hallan relacionadas con los factores de personalidad del modelo de los Cinco Factores de Costa y McCrae a la hora de enfrentarse al burnout. Según los partidarios del modelo de los Cinco Factores, la personalidad está implicada en la resolución de los problemas y en el afrontamiento del estrés. Participaron en el estudio 442 mujeres adultas de la provincia de León (España). Se les aplicaron dos instrumentos: el Ways of Coping Questionnaire (WCQ) para medir el proceso de afrontamiento y el NEO FFI para evaluar los Cinco factores de la personalidad normal. Los datos se trataron en el SPSS con análisis descriptivos y comparativos. Los resultados reflejan que las mujeres que utilizan modos de afrontamiento dirigidos a la resolución del problema son las que obtienen puntuaciones más altas en Responsabilidad, Extraversión y Apertura mental y más bajas en Neuroticismo; en tanto que las más altas en Neuroticismo utilizan fundamentalmente el modo de afrontamiento Escape.

Palabras claves: afrontamiento; mujeres; personalidad; análisis descriptivos

\section{ABSTRACT}

Factors of personality and struggles of focus on women: relations and differences. In this study we want to find out if the coping strategies formulated by Lazarus and Folkman (1986) are related to the personality traits of the model of the Five Personality Factors of Costa and McCrae. According to the followers of the Five Factors model, personality is involved in problem solving and coping with stress. 442 adult women from the province of León (Spain) participated in the study. Two instruments were applied: The Ways of Coping Questionnaire (WCQ) to measure the coping process and the NEO FFI to evaluate the Five factors of normal personality. The data were treated in 
the SPSS with descriptive and comparatives analyzes. The results show that women who use coping strategies aimed at solving the problem are those who obtain higher scores in Responsibility, Extraversion and Mental Aperture and lower scores in Neuroticism; while the higher ones in Neuroticism mainly use the Escape coping mode.

Keywords: coping women; personality; descriptive analysis

\section{INTRODUCCIÓN}

Aunque en un principio Lazarus y Folkman (1986) definieron el afrontamiento como "Ios esfuerzos cognitivos y conductuales constantemente cambiantes que se desarrollan para manejar las demandas específicas que son valoradas como situaciones que exceden o desbordan los recursos de una persona", de los trabajos posteriores realizados por Lazarus y su grupo ha quedado constancia de que estas estrategias de afrontamiento son relativamente estables en los sujetos cuando atraviesan situaciones complejas.

Consideran estos autores que cuando una persona valora o aprecia una situación como dañina, amenazante 0 de desafío, elabora inmediatamente una respuesta potencial de afrontamiento. El afrontamiento no es automático, es un patrón de respuesta susceptible de ser aprendido frente a las situaciones problemáticas.

Otra característica de su concepción es que el afrontamiento requiere un esfuerzo, unas veces consciente, otras no, pero requiere un esfuerzo dirigido a manejar la situación, que, en ocasiones, cuando no se puede cambiar, requiere un esfuerzo para adaptarse a ella.

Diferencian Lazarus y Folkman (1986) ocho modos de afrontamiento (en este estudio hemos englobado el Control y la Aceptación de la responsabilidad en el Autocontrol:

Confrontación. Describe este modo de afrontamiento los esfuerzos activos, acciones directas para alterar la situación.

Planificación. Se intenta la resolución del problema mediante una aproximación de análisis de ese problema y planificación de la respuesta.

Búsqueda de apoyo social. Describe los esfuerzos por buscar apoyo. Puede ser un apoyo social por razones más instrumentales (apoyo de información y consejo), o bien por razones emocionales (apoyo de simpatía y comprensión).

Distanciamiento. Describe los esfuerzos para separarse de la situación, olvidarse del problema, no darle importancia ni tomarlo en serio, actuar de manera que parezca que nada ha ocurrido con alusión a la creación de un punto de vista positivo.

Escape, evitación. Describe el pensamiento desiderativo que sugiere huída y evitación, se utiliza cuando el sujeto espera que ocurra un milagro que solucione el problema, trata de evitar el contacto con la gente 0 se emborracha o consume drogas para olvidar.

Autocontrol. A este modo de afrontamiento se ha unido la "Aceptación de la responsabilidad". Engloba ahora el control y la aceptación de la responsabilidad. Es decir, los esfuerzos por regular los propios sentimientos, no compartir el problema con los demás, procurar no precipitarse en las acciones, con el reconocimiento de la participación personal en el problema, disculpándose y auto criticándose.

Reevaluación positiva. Este estilo describe los esfuerzos por dar un significado positivo a la situación, potenciando desde ella el desarrollo personal.

Los partidarios del modelo de los Cinco Factores consideran que la personalidad tiene cinco dimensiones, Cinco Factores que denominan Neuroticismo, Extraversión, Apertura mental 0 Apertura a la experiencia, Amabilidad y Responsabilidad.

Los factores de este modelo son los que vamos a describir a continuación. 
Neuroticismo. Es la dimensión de la emocionalidad, es la tendencia general a experimentar emociones negativas tales como miedo, melancolía, vergüenza, ira, culpabilidad y repugnancia. Es también la propensión a tener ideas irracionales, a ser menos capaces de controlar los impulsos y enfrentarse peor que los demás con el estrés.

Extraversión. Es la dimensión de la sociabilidad. Es la preferencia por participar en grupos y reuniones. Los extravertidos son asertivos, activos y habladores. Les gusta la excitación y la estimulación y tienden a ser de carácter alegre. Son animosos, enérgicos y optimistas. Los introvertidos por el contrario son reservados, independientes, constantes. No sufren necesariamente ansiedad social. $Y$ aunque no poseen el exuberante entusiasmo de los extravertidos, no se sienten desdichados 0 pesimistas.

Apertura. Los componentes son: Integración activa, sensibilidad estética, preferencia por la variedad, curiosidad intelectual e independencia de juicio. Las personas abiertas se ven enriquecidas por la experiencia. Las personas cerradas son convencionales en su comportamiento y de apariencia conservadora; prefieren lo familiar a lo novedoso y sus respuestas emocionales son en cierto modo apagadas.

Amabilidad es la dimensión de las tendencias interpersonales. Son características de las personas amables el altruismo, la confianza, la franqueza, actitudes conciliadoras, la modestia; sienten simpatía con los demás, tienen disposición a ayudar a los demás y creen que los otros se sienten igualmente satisfechos de hacer esto mismo. La persona menos amable es egocéntrica, suspicaz respecto a las intenciones de los demás y más bien opositora que cooperadora.

Responsabilidad. En la base de esta dimensión está el auto-control, el cual puede referirse a un proceso más activo de planificación, organización y ejecución de las tareas y las diferencias individuales en esta tendencia están en la base de la Responsabilidad. Puntuaciones altas indican perseverancia, tenacidad y decisión. Algunos autores denominan a este factor como Voluntad de logro. Son responsables personas con competencia, sentido del deber y disciplina.

En este estudio deseamos averiguar si los modos de afrontamiento formulados por Lazarus y Folkman (1986) se hallan relacionados con los rasgos de personalidad del modelo de los Cinco Factores de personalidad de Costa y McCrae (1985).

\section{MÉTODO}

\section{Muestra}

Participaron en este estudio 442 de mujeres, 156 eran estudiantes universitarias y 286 amas de casa, de la provincia de León. La media de edad fue de 44 años y el rango entre 18 y 67.

\section{Instrumentos}

Se aplicó el WCQ, Cuestionario de Modos de Afrontamiento de Lazarus y Folkman que evalúa los siguientes modos de afrontar el estrés: Confrontación, Distanciamiento, Autocontrol, Aceptación de la responsabilidad, Búsqueda de apoyo social, Escape- evitación, Planificación y Reevaluación positiva.

Los Cinco Factores de la personalidad normal se estudian con el Cuestionario NEO-FFI de Costa y McCrae (1999). Los factores estudiados son: Neuroticismo versus Estabilidad emocional, Extraversión, Apertura a la experiencia, Amabilidad y Responsabilidad.

\section{Procedimiento}

Para hallar la influencia de la personalidad en los modos de afrontamiento vamos a ver las diferencias estadísticamente significativas entre las estudiantes en los factores de personalidad y en los 
modos de afrontamiento. Para ello vamos a comparar aquellas que puntúan por debajo del percentil 25 (grupo 1) y las que puntúan por encima del percentil 75 (grupo 2) en personalidad y, a través de la prueba $t$, veremos cuales son los modos de afrontamiento más utilizados por cada grupo y sus diferencias significativas.

\section{RESULTADOS}

\section{Modos de afrontamiento y personalidad en universitarias}

En la Tabla 1 se muestran los valores percentiles $(25,50$ y 75$)$ de los Cinco Factores de la personalidad con los cuales realizamos tres grupos, 1,2 y 3 . para este estudio vamos a comparar el grupo 1 que incluye a los sujetos que están por debajo del percencil 25 y el grupo 3, por encima del percentil 75. la comparación se hace a través de la prueba $t$ que nos proporciona la comparación de las medias del grupo 1 y el grupo 3.

Tabla 1. Percentiles de las puntuaciones del NEO-FFI

\begin{tabular}{c|c|c|c|c|c|c|} 
& Percentiles & Neuroticismo & Extraversión & Apertura & Amabilidad & Responsab. \\
\hline Grupo 1 & 25 & 15 & 27 & 24 & 27 & 27 \\
\hline Grupo 2 & 50 & 22 & 32 & 28 & 31 & 31 \\
\hline Grupo 3 & 75 & 26 & 37 & 33 & 34 & 35 \\
\hline
\end{tabular}

Una vez hallados los percentiles 25 y 75 vamos a comparar a las estudiantes que tienen menor Neuroticismo (puntuaciones inferiores al percentil 25 en el Cuestionario NEO- FFI) y que constituyen el grupo 1, con las que tienen mayor Neuroticismo en el grupo 2 (puntuaciones superiores al percentil 75) en los modos de afrontamiento.

Vemos en la Tabla 2 que las estudiantes que puntúan alto en Neuroticismo (percentil 75 y superior) utilizan principalmente el modo de afrontamiento Escape $(t=-2,733 ; p=, 008)$ y las que puntúan bajo en Neuroticismo los modos que utilizan y que muestran diferencias significativas son Planificación $(t=3,637 ; p=, 001)$, Distanciamiento $(t=2,913 ; p=, 005)$ y Confrontación $(t=2,697$; $p=, 009)$ por este orden de importancia. 
Tabla 2. Diferencia en modos de afrontamiento entre el grupo 1 (menor Neuroticismo) y el grupo 2 (mayor Neuroticismo)

\begin{tabular}{|c|c|c|}
\hline Modos de afrontamiento & $t$ & $\mathrm{p}$ \\
\hline Autocontrol &,- 989 & ,326 \\
\hline Búsqueda de apoyo social &,- 348 & ,729 \\
\hline Confrontación & 2,697 & 009 \\
\hline Distanciamiento & 2,913 &, 005 \\
\hline Escape & $-2,733$ & ,008 \\
\hline Planificación & 3,637 & 001 \\
\hline Reevaluación positiva &,- 883 & ,380 \\
\hline
\end{tabular}

Si comparamos las estudiantes con menor Extraversión (grupo 1) con las que tienen más Extraversión (grupo 2) a través de la prueba $t$ hallamos los siguientes resultados que se reflejan en la siguiente Tabla 3. 
Tabla 3. Diferencia en modos de afrontamiento entre el grupo 1 menor extraversión) y el grupo 2 (mayor extraversión)

\begin{tabular}{l|c|c|c|}
\multicolumn{1}{c|}{ Modos de afrontamiento } & $t$ & $\mathrm{p}$ & \\
\hline Autocontrol &, 559 &, 578 & \\
\hline Búsqueda de apoyo social & $-2,771$ &, 007 & \\
\hline Confrontación & $-2,266$ &, 026 & \\
\hline Distanciamiento & $-1,597$ &, 115 & \\
\hline Escape & 1,972 &, 052 & \\
\hline Planificación & $-4,779$ &, 000 & \\
\hline Reevaluación positiva &,- 263 &, 793 & \\
\hline
\end{tabular}

Variable independiente: EXTRAVERSIÓN. Frecuencia: N1 = 37; N2 = 38

En la Tabla 3 se muestran las diferencias entre las estudiantes con mayor y menor nivel de Extraversión. Las diferencias se manifiestan en que los modos Planificación, con puntuaciones $t=$ 4,$779 ; p=, 000$, en Búsqueda de apoyo social $(t=2,771 ; p=, 007)$ y Confrontación $(t=2,266 ; p=$ ,026) que son más utilizados por las estudiantes más Extravertidas, en tanto que las introvertidas utilizan más el modo Escape $(t=1,972 ; p=, 052)$.

Cuando queremos saber qué modos de afrontamiento utilizan las estudiantes menos abiertas (Grup01) frente a las más abiertas (Grupo 2) vemos los resultados reflejados en la siguiente Tabla 4 que refleja las diferencias entre las estudiantes con mayor nivel de Apertura (grupo 2) y las que tienen menor nivel de Apertura (grupo 1). Las diferencias se manifiestan en que las del grupo 2 utilizan más el modo Planificación $(t=-3,462 ; p=, 001)$, el modo Búsqueda de apoyo social $(t=-2,847$; $p=, 006)$, el modo Confrontación $(t=-2,709 ; p=, 008)$ y la Reevaluación positiva $(t=-2,449 ; p=$ ,017). 
Tabla 4. Diferencia en modos de afrontamiento entre el grupo 1 (menor Apertura) y el grupo 2 (mayor Apertura)

\begin{tabular}{|c|c|c|}
\hline Modos de afrontamiento & $t$ & $\mathrm{p}$ \\
\hline Autocontrol & $-1,107$ & ,272 \\
\hline Búsqueda de apoyo social & $-2,847$ &, 006 \\
\hline Confrontación & $-2,709$ &, 008 \\
\hline Distanciamiento &, 144 & ,886 \\
\hline Escape & 1,036 & ,304 \\
\hline Planificación & $-3,462$ &, 001 \\
\hline Reevaluación positiva & $-2,449$ &, 017 \\
\hline
\end{tabular}

Variable independiente: APERTURA. Frecuencia: N1 = 38; N2 = 37

¿Qué modos de afrontamiento utilizan preferentemente las estudiantes según el factor Amabilidad? 
Tabla 5. Diferencias en modos de afrontamiento entre el grupo 1 (menor Amabilidad) y el grupo 2 (mayor AMABILIDAD)

\begin{tabular}{l|c|c|c|} 
Modos de afrontamiento & $\mathrm{t}$ & $\mathrm{p}$ & \\
\hline Autocontrol &,- 751 &, 455 & \\
\hline Búsqueda de apoyo social & $-1,590$ &, 115 & \\
\hline Confrontación &,- 021 &, 983 & \\
\hline Distanciamiento &, 712 &, 478 & \\
\hline Escape & 2,379 &, 019 & \\
\hline Planificación &,- 334 &, 739 & \\
\hline Reevaluación positiva &,- 792 &, 430 & \\
\hline
\end{tabular}

Variable independiente: AMABILIDAD. Frecuencia: N1 = 49; N2 $=43$

La Tabla 5 nos muestra la diferencia entre las estudiantes que tienen más Amabilidad (Grupo 2) y menos amabilidad (Grupo1) y los modos de afrontamiento preferidos. Las que tienen más Amabilidad se diferencian por hacer mayor uso de Escape $(t=2,379 ; p=, 019)$.

Los modos de afrontamiento que diferencian a las estudiantes altas en Responsabilidad (Grupo 2) de las más bajas en este factor (Grupo1) se reflejan en la Tabla 6.

La Tabla 6 muestra las diferencias significativas entre las estudiantes. Las que tienen mayor nivel de responsabilidad utilizan preferentemente los modos Planificación con puntuaciones $t=4,982 ; p$ $=, 000$ y Confrontación $(t=3,057 ; p=, 003)$. Las menos responsables utilizan preferentemente el Escape $(t=2,626 ; p=, 010)$. 
Tabla 6. Diferencias en modos de afrontamiento entre el grupo 1 (menor Responsabilidad) y el grupo 2 (mayor Responsabilidad).

\begin{tabular}{l|c|c|c|}
\cline { 4 - 5 } Modos de afrontamiento & $t$ & $\mathrm{p}$ & \\
\hline Autocontrol &, 247 &, 805 & \\
\hline Búsqueda de apoyo social & $-1,903$ &, 060 & \\
\hline Confrontación & $-3,057$ &, 003 & \\
\hline Distanciamiento & 1,168 &, 246 & \\
\hline Escape & 2,626 &, 010 & \\
\hline Planificación & $-4,982$ &, 000 & \\
\hline Reevaluación positiva &,- 467 &, 642 & \\
\hline
\end{tabular}

Variable independiente: RESPONSABILIDAD. Frecuencia: N1 = 43; N2 $=46$

Vamos a ver a continuación las diferencias entre las estudiantes universitarias y las amas de casa en los modos de afrontamiento que utilizan más frecuentemente. La Tabla 7 muestra que las estudiantes prefieren la Búsqueda de apoyo social a la hora de afrontar los problemas que les producen estrés, en tanto que las amas de casa prefieren la Reevaluación positiva $(t=5,039 ; p=, 000)$ y la Confrontación $(t=3,286 ; p=024)$. 
Tabla 7. Diferencias en modos de afrontamiento entre estudiantes (mujeres) y amas de casa

\begin{tabular}{l|c|c|}
\multicolumn{1}{c|}{ Modos de afrontamiento } & $\mathrm{t}$ & $\mathrm{p}$ \\
\hline Autocontrol &,- 822 &, 411 \\
\hline Búsqueda de apoyo social & 3,286 &, 001 \\
\hline Confrontación & $-2,272$ &, 024 \\
\hline Distanciamiento & $-1,428$ &, 154 \\
\hline Escape & $-1,409$ &, 159 \\
\hline Planificación &,- 578 &, 563 \\
\hline Reevaluación positiva & $-5,039$ &, 000 \\
\hline
\end{tabular}

Frecuencia: $\mathrm{N}$ amas de casa $=286 ; \mathrm{N}$ estudiantes $=156$

\section{DISCUSIÓN Y CONCLUSIONES}

Lazarus y Folkman han dejado claro en sucesivas publicaciones que todas las personas utilizamos un amplio abanico de estrategias de afrontamiento y que los mecanismos particulares de afrontamiento elegidos en una situación determinada dependen de múltiples factores, especialmente de las exigencias y oportunidades de la situación. Pero, aunque el afrontamiento no es una expresión directa de la personalidad, está ciertamente influido por rasgos de la misma (Costa y McCrae, 1984, 1989). Estos autores han hallado que muchos mecanismos de afrontamiento, teóricamente inmaduros o neuróticos (fantasías de evitación, estados de indecisión, uso de sustancias, auto- culpabilidad y reacciones hostiles), se relacionan significativamente con las puntuaciones del factor Neuroticismo del NEO-PIR. También hallaron similares efectos en los factores Extraversión y Apertura. La Extraversión se relaciona con el uso del pensamiento positivo, acción racional y moderación. Las personas altas en el factor Apertura utilizan el humor al enfrentarse a problemas, mientras que las bajas en Apertura se apoyan más en la fe. Otro estudio (Costa, Zonderman y McCrae, 1991) analizó las relaciones entre los Cinco factores y tres conjuntos de medidas de mecanismos de defensa. En él se encontró que Neuroticismo está relacionado con regresión, desplazamiento, duda y patrones inadaptados de acción, lo que confirma la asociación de Neuroticismo con estilos 
inadecuados de afrontamiento. Extraversión se relacionó positivamente con negación y negativamente con duda, reflejando la auto- seguridad de los extravertidos. Apertura se relacionó positivamente con las defensas adaptativas mientras que Amabilidad lo hizo negativamente con las defensas distorsionantes de la imagen (o superioridad) y positivamente con las defensas de auto- sacrificio. Responsabilidad, por último, se relacionó positivamente con protagonismo y negativamente con regresión y formas inadaptadas de actuación, lo que sugiere un estilo de afrontamiento maduro como ha sido descrito por Vickers, Kolar y Hervig (1989). McCrae y Costa (1996) también hallaron relaciones significativas entre Neuroticismo y el uso creciente de las estrategias de afrontamiento centradas en la emoción, en tanto que la Extraversión se relacionó con estrategias centradas en el problema y la Reevaluación positiva.

En nuestro estudio los hallazgos encontrados en los modos de afrontamiento, su relación con las variables de personalidad y las diferencias entre los grupos son los siguientes (ver Tabla 8).

Las universitarias con Neuroticismo alto utilizan más frecuentemente el modo de afrontamiento Escape en tanto que las más estables emocionalmente (Neuroticismo bajo) prefieren hacer uso de Ios modos Planificación, Distanciamiento y Confrontación, por este orden de importancia.

Los modos de afrontamiento elegidos por las universitarias que puntúan más alto en Extraversión son Planificación, Búsqueda de apoyo social y Confrontación en tanto que el modo más utilizado por las universitarias más introvertidas es Escape.

Cuando comparamos por Apertura hallamos que quienes puntúan alto en este factor utilizan preferentemente los modos de afrontamiento Planificación, Búsqueda de apoyo social, Confrontación y Reevaluación positiva, por este orden.

Las universitarias que puntúan más alto en Amabilidad se caracterizan porque a la hora de afrontar los problemas utilizan preferentemente el Escape en mayor medida que las que puntúan bajo.

El factor Responsabilidad diferencia a las universitarias altas de las bajas en que las primeras hacen uso fundamentalmente de los modos Planificación y Confrontación, en tanto que las segundas, las más bajas en este factor utilizan fundamentalmente el Escape cuando se enfrentan a situaciones estresantes.

Las estudiantes universitarias se diferencian de las amas de casa en que utilizan más frecuentemente el modo de afrontamiento Búsqueda de apoyo social en tanto que las amas de casa prefieren los modos Reevaluación positiva y Confrontación.

Los modos de afrontamiento centrados en el problema como Planificación y Confrontación son los más frecuentemente utilizados por las universitarias con mayor equilibrio emocional (Neuroticismo Bajo), altas en Extraversión, Apertura y Responsabilidad.

El modo Escape es el elegido por las universitarias altas en Neuroticismo y Amabilidad y bajas en Extraversión y Responsabilidad. El modo Distanciamiento es más utilizado por las universitarias bajas en Neuroticismo.

Búsqueda de apoyo social es mas utilizado por las universitarias con puntuaciones altas en Apertura y en Extraversión.

Reevaluación positiva es el modo más valorado por las universitarias con altas puntuaciones en Apertura.

Amas de casa y estudiantes universitarias se diferencian a la hora de afrontar problemas con estrés en que estas últimas prefieren la Búsqueda de apoyo social mientras que las amas de casa se enfrentan más activamente a los problemas (Confrontación) y también hacen más uso de la Reevaluación positiva. 


\section{REFERENCIAS BIBLIOGRÁFICAS}

Costa, P. T. y McCrae, R. R. (1985). The NEO Personality Inventory manual. Odessa, FL: Psychological Assessment Resources.

Costa, P. T. y McCrae, R. R. (1984). Personality as a life-long determinant of web- being. En C. Malatesta y C. Izard (Eds.), Affective process in adult development and aging, (141-157). Beverly Hills, CA: Sage.

Costa, P. T. y McCrae, R. R. (1989). Personality, stress and coping: Some lessons from a decade of research. En K. S. Markides y C. L. Cooper (Eds.), Aging, stress, social support and health, (267-283). Nueva York: Wiley.

Costa, P. T. y McCrae, R. R. (1999). NEO-PI-R, Inventario de Personalidad NEO Revisado. NEOFFI, Inventario NEO reducido de Cinco Factores. Madrid: TEA Ediciones.

Costa, P. T., Somerfield, M. R. y McCrae, R. R. (1996). Personality and coping: A reconceptualization. En M. Zeidner y N. Endler (Eds.), Handbook of coping. Theory, research, applications, (4461). Nueva York: Wiley and Sons.

Costa, P. T., Zonderman, A. B. y McCrae, R. R. (1991). Personality, defense, coping, and adaptation in older adulthood. En E. M. Cummings, A. L. Greene y K. H. Karraker (Eds.), Life-span developmental Psychology: Perspectives on stress and coping, (277-293). Hillsdale, NJ: Lawrence Erlbaum Associates

Goldberg, L. R. (1992). The development of markers of the Big-Five factor structure. Psychological Assessment, 4, 26-42.

Hewitt, P. L. y Flett, G. L. (1996). Personality traits and the coping process. En M. Zeidner y N. S. Endler, Handbook of coping: Theory, research, applications (pp. 410-433). Nueva York: Wiley.

Lazarus, R. S. y DeLongis, A. (1983). Psychological stress and coping in aging. American Psychologist, 38, 245-254.

Lazarus, R. S. y Folkman, S. (1986). Estrés y procesos cognitivos. Barcelona: Martínez Roca. (orig. de 1984).

McCrae, R. R. y Costa, P. T. (1986). Personality, coping and coping effectiveness in an adult simple. Journal of Personality, 54, 385-405.

Maslach, C. y Jackson, S. E. (1986). Maslach Burnout Inventory. Manual (2ª ed.). Palo Alto, CA: Consulting Psychologists Press.

Maslach, C. y Jackson, S. E. (1997). MBI. Inventario "Burnout" de Maslach. Madrid: TEA Ediciones.

Maslach, C. y Pines, A. (1977). The burnout syndrome in the day care setting. Child Care Quarterly, 6, 100-113.

Maslach, C., Schaufeli, W. B. y Leiter, M. P. (2001). Job Burnout. Annual Review of Psychology, 52, 397-422.

Sandín, B. (1996). El Estrés. En A. Belloch, B. Sandín y F. Ramos. Manual de Psicopatología, vol. 2 (pp. 3-52). Madrid: McGraw-Hill.

Vickers, R. R., Jr.; Kolar, D. W. y Hervig, L. K. (1989). Personality correlates of coping with military basic training (Inform $\mathrm{n}^{\circ} 3$ ). San Diego, CA: Naval Health Research Center. 